\title{
O MODELO dOS “CINCO DOMÍNIOS” DO BEM-ESTAR ANIMAL APLICADO EM SISTEMAS INTENSIVOS DE PRODUÇÃO DE BOVINOS, SUÍNOS E AVES
}

\author{
Janaina da Silva Braga, Fernanda Macitelli, Victor Abreu e lima \& Taciana Diesel
}

\begin{abstract}
'Programa de Pós-graduação em Zootecnia, Faculdade de Ciências Agrárias e Veterinárias, Universidade Estadual Paulista, Jaboticabal, 14884-900, SP, Brasil.

${ }^{2}$ Instituto de Ciências Agrárias e Tecnológicas, Universidade Federal do Mato Grosso, Rondonópolis, 78735-910, MT, Brasil. ${ }^{3}$ Departamento de Zootecnia, Universidade Estadual de Santa Catarina, Chapecó, 89815-630, SC, Brasil.

${ }^{4}$ Grupo de Estudos e Pesquisas em Etologia e Ecologia Animal, Departamento de Zootecnia, Faculdade de Ciências Agrárias e Veterinárias, Universidade Estadual Paulista, Jaboticabal 14884-900, SP, Brasil.
\end{abstract}

\begin{abstract}
The Five Domains model of animal welfare applied in intensive animal production systems. A demand for intensive production systems is a global reality with a tendency to grow in the coming decades. However, these systems have limitations in terms of care as physical, behavioral and psychological needs of animals, which can lead to the impoverishment of their welfare. Although animal welfare has already been widely defined, its evaluation within animal production still poorly applied. Among the forms of evaluation of animal welfare, we can consider the "Five Domains" model, proposed initially by MELLOR \& REID (1994). This model is a systematic method that includes four physical or functional domains (Nutrition, Environment, Health and Behavior) and a mental domain (Mental or Affective State). This article begins briefly describing the features of the "Five Domains" model. In order to illustrate the possible interactions between the five animal welfare domains, three recognizable situations that affect the welfare of production animals are presented: reduced space allowance at beef feedlot, painful handling of piglets and transport of broilers. In this context, although it is not possible to describe all the intervening factors and their possible combinations that potentially affect animal welfare, we believe that the examples presented showed an integrated view on the risks of compromising the welfare of animals in intensive production systems.
\end{abstract}

Keyword: high density on transport, reduced space allowance at feedlot, tail docking, teeth clipping.

Resumo. A demanda por sistemas intensivos de produção é uma realidade mundial com tendência de crescimento nas próximas décadas. No entanto, esses sistemas apresentam limitações em atender as necessidades físicas, comportamentais e psicológicas dos animais, o que pode acarretar no empobrecimento do bem-estar dos mesmos. Apesar do bem-estar animal já ser amplamente definido, sua avaliação dentro da produção animal ainda é pouco aplicada. Dentre suas formas de avaliação, o modelo dos "Cinco Domínios", proposto por MelLoR \& REID (1994), atua como um método sistemático que inclui quatro domínios físicos ou funcionais (Nutrição, Ambiente, Saúde e Comportamento) e um domínio mental (Estado Mental ou Afetivo). Este artigo descreve sucintamente as características do modelo "Cinco Domínios" e exemplifica as possíveis interações entre os domínios em três situações reconhecidamente críticas para bem-estar dos animais de produção: restrição de espaço no confinamento de bovinos, procedimentos dolorosos no manejo de leitões e alta densidade no carregamento e transporte de frangos de corte. Nesse contexto, apesar de não ser possível incluir todos os fatores intervenientes nessa dinâmica, os exemplos apresentados mostram uma visão integrada sobre os riscos de comprometimento do bem-estar dos animais em sistemas intensivos de produção. 
Palavras-chave: alta densidade no transporte, caudectomia, corte de dente, restrição de espaço em confinamento.

\section{INTRODUÇÃO}

Após a segunda guerra mundial, a crescente demanda por proteína de origem animal aliada às pressões econômicas incentivaram as cadeias produtivas de carne a adotarem sistemas intensivos, resultando em aumento da produtividade e redução do espaço utilizado (VANHONACKER et al., 2009). São inegáveis os benefícios econômicos advindos desses sistemas e, a demanda para os mesmos se tornarem ainda mais eficientes e sustentáveis continuará crescente nas próximas décadas (GARNETT et al., 2013).

As análises econômicas, principalmente as que envolvem a lucratividade, são o foco dos sistemas produtivos, mas raramente levam em consideração o quanto se deixa de ganhar em sistemas onde os animais enfrentam dificuldades para se adaptar a ambientes que não atendem às suas necessidades físicas, comportamentais e psicológicas (FRASER, 1983). As tentativas de adaptar-se continuamente a um ambiente desafiador resultam em alto custo biológico para o animal, e risco de comprometimento dos índices produtivos. Assim, os possíveis conflitos entre os sistemas produtivos e as necessidades dos animais podem ser minimizados pelos benefícios econômicos advindos da melhoria no bem-estar dos animais, como por exemplo redução da taxa de morbidade e mortalidade, melhoria do status de saúde com maior resistência a doenças, menor uso de medicamentos, menor risco de zoo- noses e de doenças transmitidas por alimentos.

Adicionalmente, o bem-estar de animais de produção está diretamente relacionado à qualidade dos produtos de origem animal (BLOKHUIS et al., 2003 e 2008; De PAsSiLlé \& RUSHEN, 2005; VANHONACKER et al., 2009), principalmente nas sociedades onde sustentabilidade e ética são o centro das discussões políticas e públicas sobre os sistemas de produção (VermeIr \& VERBEKE, 2006). É notório que o bem-estar animal vem se tornando cada vez mais importante dentro do cenário da produção animal e, para que este possa ser discutido e avaliado, é necessária uma definição clara, além de métodos objetivos, validados cientificamente. A definição mais aceita, proposta por BROOM (1986), considera que bem-estar animal é o estado de um indivíduo em relação as suas tentativas em se adaptar ao meio em que vive. Expandindo essa definição, a OIE (2013) afirma que bem-estar animal é como um animal responde às condições em que vive, sendo considerado alto grau de bem-estar quando está bem nutrido, saudável, confortável, seguro, capaz de expressar seu comportamento natural, e se não estiver sentindo dor, medo ou angústia. Isso implica em nutrição, sanidade e ambiente adequados, tratamento veterinário, boas práticas de manejo e abate humanitário.

Os protocolos de avaliação de bem-estar animal normalmente utilizam medidas padronizadas e objetivas para os critérios de avaliação, 
incluindo nutrição, sanidade, ambiente, expressão de comportamentos naturais e sentimentos (Welfare QUALITY', 2009; AWIN, 2015ab), primariamente baseadas nos animais e no ambiente e depois integradas em um modelo geral de avaliação. Nesse contexto, o modelo dos "Cinco Domínios" do bem-estar animal, proposto por MELLOR \& REID (1994), apresenta-se como um método sistemático, estruturado e abrangente de avaliação o bem-estar dos animais. Esse modelo funciona como uma ferramenta de avaliação e gerenciamento de bem-estar animal e não deve ser considerado como uma representação fidedigna da relação entre estrutura e função do organismo animal, tampouco como uma definição de bem-estar animal. O modelo considera quatro domínios que contemplam os estados internos ou físico-funcionais do animal, sendo eles "Nutrição" (Domínio 1), "Ambiente" (Domínio 2), "Saúde" (Domínio 3) e "Comportamento" (Domínio 4). O comprometimento dos domínios físicos (Domínios 1 a 4) é usado para inferir cautelosamente quaisquer experiências afetivas associadas ao domínio "Mental" (Domínio 5). Recentemente, esse modelo foi atualizado com a inclusão dos estados mentais positivos (MELLOR \& BEAUSOLEIL, 2015; MelloR, 2016; MELLOR, 2017).

As principais características do modelo apresentadas na Figura 1 são amplamente descritas na literatura científica, mas destaca-se que em algumas espécies essas características podem ser inapropriadas. Dessa maneira, os usuários do modelo são encorajados a incluir ou excluir fatores, levando em consideração o com- portamento natural, a biologia e a ecologia da espécie em questão, além da respectiva relação com o ambiente social e físico sob avaliação. 0 organismo funciona como uma entidade dinâmica e integrada, ou seja, à medida que as funções ou estados internos do corpo, circunstâncias externas e estados mentais se relacionam, inevitavelmente ocorrem interações entre os domínios, que podem ser caracterizadas como relações de causa e efeito.

Cada avaliação do estado geral de bem-estar dos animais dentro desse modelo pode ser considerada hipotética na medida em que as inferências são feitas (MELLOR, 2017). Entretanto, todas elas devem ser baseadas no conhecimento científico, incluindo principalmente as áreas da fisiologia, neurofisiologia, neurociência afetiva e etologia. Um dos principais benefícios dessa abordagem é que ela permite separar claramente os impactos físicos e/ou funcionais (Domínios 1 a 4) dos estados mentais ou afetivos dos animais (Domínio 5) que, em última instância, determinam o status de bem-estar do animal (Figura 1). Ainda, essa abordagem destaca a importância dos animais experimentarem estados mentais positivos, considerando-os como elementos importantes na definição do status de bem-estar dos animais. Destaca-se que os animais de produção são capazes de experimentar conscientemente estados mentais negativos e positivos, associados às limitações ou aos atendimentos das necessidades físicas e/ou funcionais, respectivamente (MELLOR et al., 2009). Para informações mais detalhadas desse modelo e suas aplicações em diferentes 


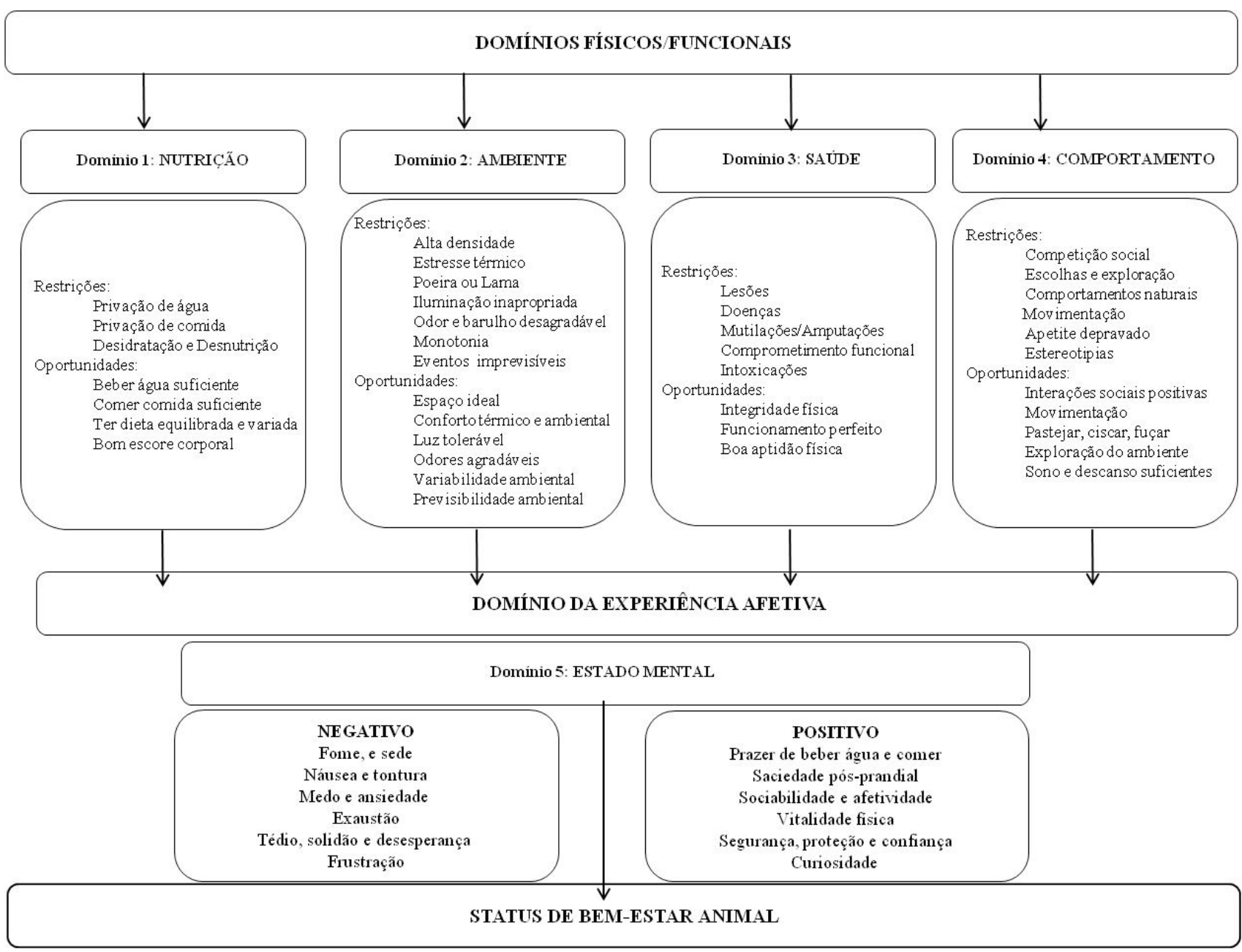

Figura 1. Modelo “Cinco Domínios" do bem-estar animal, adaptado de MELLOR \& BEAUSOLEIL (2015).

contextos, ver BEAUSOLEIL et al. (2012) e BEAUSOLEIL \& MELLOR (2015).

De maneira geral, o uso de estados mentais positivos ainda é limitado no cenário da produção animal. Assim, os modelos que serão discutidos a seguir, seguem a estrutura inicial apresentado por MELLOR \& REID (1994), e apresentam três situações reconhecidamente limitantes para bem-estar dos animais de produção: a) restrição de espaço no confinamento de bovi- nos, b) procedimentos dolorosos no manejo de leitões e, c) alta densidade no carregamento e transporte de aves.

\section{Aplicação do modelo "Cinco Domínios" para AVALIAÇÃO DO BEM-ESTAR DE BOVINOS EM CONFI- NAMENTO COM REDUÇÃO DO ESPAÇO DISPONÍVEL POR ANIMAL}

O Brasil é detentor de posição de destaque no cenário global de produção e exportação de carne bovina, onde tem sido notória a cres- 
cente intensificação da bovinocultura de corte, principalmente pela utilização de confinamentos para a terminação de bovinos (RASMUSSEN et al., 2014). De acordo com MiLLEN et al. (2009), o confinamento de bovinos de corte minimiza os efeitos da baixa disponibilidade de forragem no período seco do ano, da pressão sobre os recursos naturais (PELLetier et al., 2010), da competição das culturas de grãos por área de terras agrícolas (RASMUSSEN et al., 2014), além de ser uma das estratégias para aumentar a produtividade por área, atendendo à crescente demanda externa por carne bovina de qualidade (OLIVEIRA \& MiLLEN, 2014).

Confinar, de acordo com a premissa básica dessa atividade, implica na redução do espaço disponível por animal e na dependência total do homem para se alimentar. Apesar dos confinamentos no Brasil apresentarem ciclos curtos, entre 70 e 90 dias (MILLEN et al., 2009), estudos recentes têm demonstrado que a redução no espaço disponível em confinamento ao ar livre em condições brasileiras (MACITELLI, 2015), similarmente aos confinamentos "indoor" americanos e canadenses (FISHER et al., 1997; HICKEY et al., 2003; GUPTA et al., 2007), implicam em potenciais impactos negativos sobre o bem-estar dos animais, quando comparados aos sistemas de produção de bovinos mantidos em pastagens (LEE et al., 2013). Esse fato ocorre, possivelmente, devido ao fato dos sistemas intensivos serem muito diferentes do ambiente no qual o bovino foi evolutivamente preparado e adaptado para viver (RUSHEN \& DE PASSILÉ, 1992; BROOM \& FRA-
SER, 2007; PHILLIPS, 2008).

Transferir animais para o ambiente de confinamento implica na imposição de diversos fatores ambientais como: mudança na dieta e no regime alimentar, reagrupamento social, maior exposição aos patógenos, bem como exposição a condições climáticas extremas (FELL et al., 1998; MADER, 2003). Esses fatores estimulam a ativação de mecanismos adaptativos, em busca da manutenção da homeostase (MORMÈdE et al., 2007), ocasionando mudanças comportamentais, metabólicas e endócrinas que podem ser vistas ao longo do tempo de confinamento. Como descrito por VEISSIER \& BOISSY (2007), essa situação, se persistente, estabelece-se em posição oposta à definição operacional de alto grau de bem-estar animal proposta por BRoom (1986), uma vez que as muitas falhas nas tentativas em adaptar-se ao ambiente de restrição de espaço implicam em elevados custos biológicos para o animal, o que pode ser evidenciado pela redução do ganho de peso vivo, da taxa de ingestão e da eficiência alimentar (INGVARTSEN \& ANDERSEN, 1993; FISHER et al., 1997; FELL et al., 1999; HICKEY et al., 2003; GUPTA et al., 2007; MACITELLI, 2015).

Considerando o modelo dos "Cinco Domínios" do bem-estar animal, proposto por MELLOR \& REID (1994), a redução do espaço disponível por animal em uma baia de confinamento pode, inicialmente, ser considerada como um desafio ambiental, ou seja, compromete o Domínio 2 - Ambiente, que por sua vez tem ação sobre os demais domínios (Figura 2). 


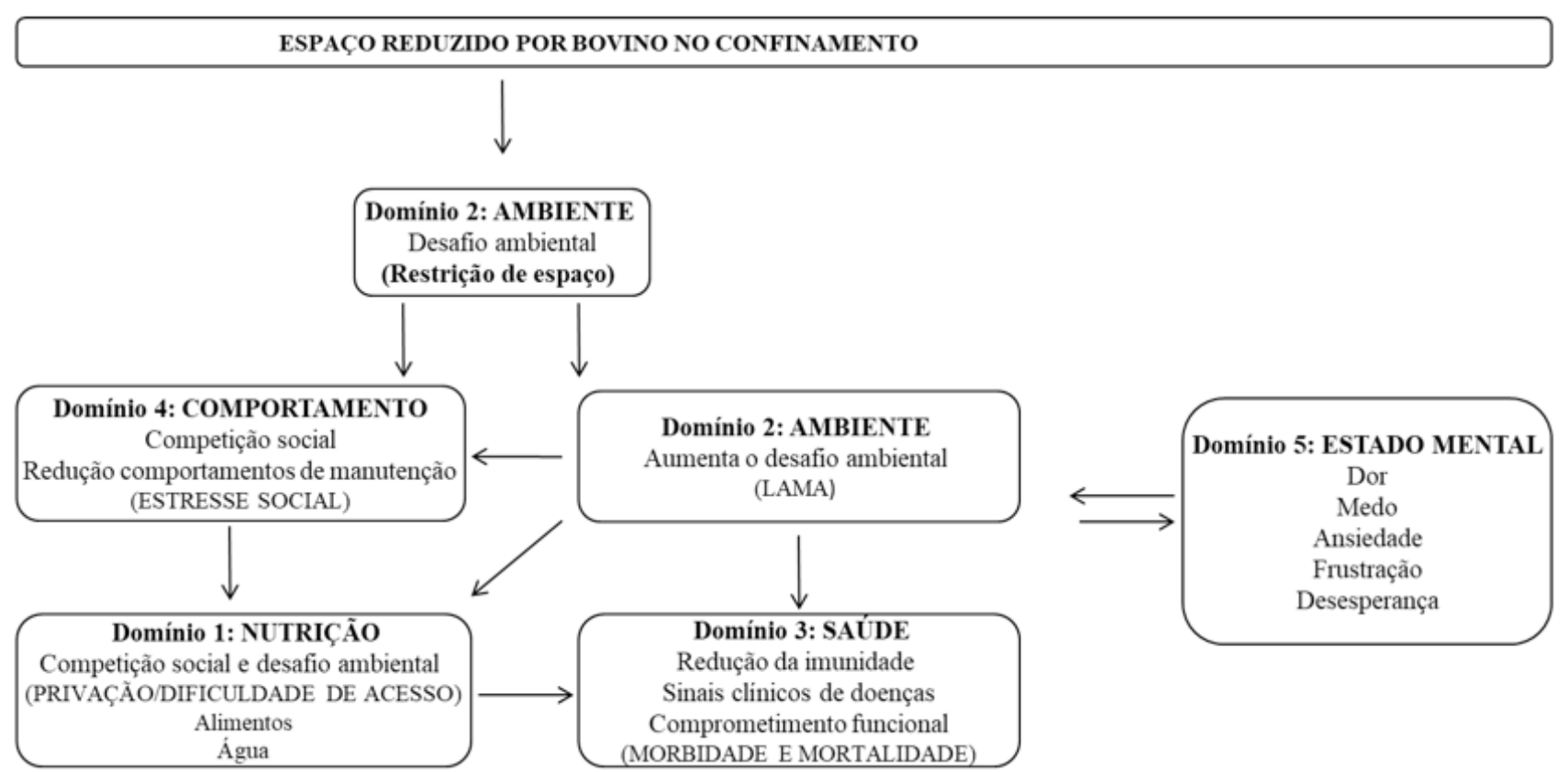

Figura 2. Aplicação do modelo "Cinco Domínios", proposto por MELLOR \& REID (1994) para a avaliação do impacto da restrição de espaço em confinamento sobre o bem-estar de bovinos, baseando-se na literatura científica.

De acordo com PARANHOS DA COSTA (2000), ambiente pode ser definido como o espaço constituído por um meio físico e psicológico, preparado para o exercício das atividades do animal que nele vive. Assim, o ambiente dos bovinos mantidos em regime de confinamento é notoriamente desafiador. Bovinos alojados sob restrição de espaço também apresentam comprometimento do Domínio 4 - Comportamento, devido ao estresse social que resulta no aumento da competição e das interações agressivas entre os membros do grupo (FRASER, 1980; Kondo et al., 1989; FISHER et al., 1997; LINDBERG, 2001). IsSo acontece porque os bovinos são animais gregários com ricos padrões de organização social que têm sua expressão limitada, parcial ou totalmente, dentro do ambiente de confinamento. Assim, a restrição de espaço em confinamento pode ser responsável por hierarquias de dominância instáveis, caracterizadas por baixo nível de previsibilidade e de controle social para os animais, resultando em fonte adicional de estresse físico e psicológico (SAPOLSKY, 2005).

Com o passar dos dias em confinamento, os animais serão fonte de pressão física nas baias, devido a sua movimentação, e de excesso de umidade (lama), devido ao acúmulo de urina e fezes, principalmente nas áreas próximas ao cocho e ao bebedouro (MADER, 2008). A declividade da baia, a condição do solo, bem como a quantidade de chuva são fatores reconhecidos como capazes de minimizar ou maximizar a formação de lama dentro das baias de confinamento (GRANDIN, 2016). A formação de lama potencializa o efeito negativo da restrição de espaço, 
afetando ainda mais o Domínio 2 - Ambiente.

Quando o acúmulo de lama na baia de confinamento atinge entre 11 e $20 \mathrm{~cm}$ de profundidade (condição considerada como moderada), ocorre uma redução entre 8 e 15\% da taxa de ingestão (SWEETEN et al., 2014) e em casos mais severos, entre 30 e $60 \mathrm{~cm}$ de profundidade (condição severa), ocorre uma redução entre 15 e $30 \%$ da taxa de ingestão, respectivamente (NRC, 1986), ou seja, a presença de lama pode afetar também o Domínio 1 - Nutrição. Considerando que bovinos não gostam de deitar-se em lugares sujos e, se tiverem a oportunidade, irão escoIher os locais mais secos para fazer isso (FISHER et al., 2003), a formação de lama também afeta o Domínio 4 - Comportamento, sendo esperada maior competição entre os animais para acesso às áreas mais secas e, dependendo da restrição, acesso privilegiado dos dominantes. Adicionalmente, sob restrição de espaço, observa-se redução no tempo de permanência deitado (FISHER et al., 1997; NAPOLITANO et al., 2004; GYGAX et al., 2007). Deitar é um comportamento de manutenção extremamente importante para o descanso e a ruminação dos bovinos. Quase $90 \%$ da atividade de ruminação é realizada quando os animais estão deitados, já que nessa posição ocorre um aumento da pressão abdominal, que facilita a ruminação tornando-a mais eficiente (SEGABINAZZI et al., 2014).

Pode-se afirmar que o excesso de lama nas baias afeta diretamente o Domínio 3 - Saúde, uma vez que torna o piso escorregadio para os animais caminharem, bem como oferece a condição de exposição crônica do casco a um ambiente úmido que compromete a barreira de proteção epitelial interdigital, predispondo a lesões e podridão nos cascos (STOKKA et al., 2001). Existem ainda outros problemas de saúde que podem estar associados à redução da disponibilidade de espaço aos bovinos e, consequentemente, piores condições ambientais, tais como pneumonia e enfisema pulmonar (MACITELLI, 2015). KRAWCZEL et al. (2012) destacaram que muitos dos problemas de saúde dos bovinos confinados surgem devido à baixa imunidade em resposta ao estresse exercido pela restrição de espaço.

Ressalta-se ainda que nas épocas mais quentes, o excesso de lama limita a habilidade do animal em dissipar calor e oferece área potencial para a reprodução de moscas hematófagas, com efeitos sobre os Domínios 1 - Nutrição, Domínio 3 - Saúde e Domínio 4 - Comportamento, uma vez que, as moscas picam os animais causando dor e perda de sangue, e evocam os comportamentos dos animais de levantar os membros, balançar a cauda e sacudir a cabeça, ambos contribuindo para o estresse térmico por calor, podendo reduzir a taxa de ingestão e o ganho de peso (CATANGul et al., 1997; CAMPBELl et al., 2001).

Como brevemente apresentado, é evidente que as tentativas do organismo animal em adaptar-se ao ambiente do confinamento com reduzida disponibilidade de espaço provocam várias alterações fisiológicas a médio e longo prazo, que podem caracterizar o estresse crônico. Sob situações de estresse crônico, os níveis de glicocorticóides circulantes são constantemente 
elevados, resultando em alterações que podem afetar diretamente a saúde e o desempenho, incluindo catabolismo proteico, hiperglicemia, atrofia do tecido linfóide, redução do número de linfócitos e anticorpos, ocasionando falhas no sistema imunológico e lesões aos tecidos e órgãos, com redução do ganho de peso e alterações psicológicas como apatia (MATTERI et al., 2000; TSIgOS \& Chrousos, 2002; CARROL \& FORSBERG, 2007).

Conforme apresentado, o comprometimento dos quatro domínios físicos/funcionais, afeta o Domínio 5 - Estado Mental, onde os animais experimentam sentimentos negativos como dor, medo, ansiedade, frustração e desesperança por não poderem se retirar do ambiente ou terem oportunidades para fazerem escolhas.

\section{Aplicação do modelo "Cinco domínios" para} AVALIAÇÃO DO BEM-ESTAR DE LEITÕES SUBMETIDOS À CAUDECTOMIA E AO CORTE OU DESGASTE DOS DENTES

Nas últimas décadas o Brasil se consolidou como um dos grandes produtores e exportadores mundiais de carne suína e abateu cerca de 10,62 milhões de animais apenas no segundo trimestre de 2017 (IBGE, 2017). Essa posição de destaque reflete os altos índices produtivos alcançados devido às melhorias no potencial genético, na sanidade e na alimentação dos animais. Adicionalmente, o setor passa por acelerado processo de automatização nos sistemas de alojamento, distribuição da alimentação e ambiência, ao mesmo tempo que enfrenta uma sé- rie de pontos críticos relacionados ao bem-estar dos animais. Entre eles as condições do manejo pré-abate, especialmente durante o transporte, o alojamento das matrizes gestantes, a falta de enriquecimento ambiental nas instalações, o desmame precoce dos leitões e os procedimentos potencialmente dolorosos de rotina como a caudectomia e o desgaste ou cortes dos dentes, aos quais os leitões são submetidos entre o primeiro e o terceiro dia de vida.

Destaca-se que a ocorrência da caudofagia aumentou com a intensificação dos sistemas de produção e pode ser considerada um dos problemas comportamentais mais comuns na suinocultura (NANNONI et al., 2014). A limitação, parcial ou total, dos suínos expressarem seu comportamento natural de explorar o ambiente tem sido relacionada com a ocorrência de caudofagia entre os animais, que redirecionam esse comportamento exploratório para a cauda dos demais membros do grupo, mordendo-as até provocar ferimentos. De acordo com uma análise de fatores de risco ligados à ocorrência de caudofagia, Scollo et al. (2013) relataram que o risco é maior em baias com alta densidade, quando os animais não possuem acesso facilitado à água e quando não há renovação do ar dentro do galpão de alojamento.

Apesar de comum na fase de creche, a caudofagia também ocorre na fase de terminação e tem sido associada com a maior ocorrência de abscessos na coluna vertebral, problemas locomotores, pulmonares e renais, o que resulta em condenações parciais ou totais das carcaças e 
das vísceras dos animais após o abate (KRITAS \& MORRISON, 2007), perdas econômicas para todo o setor (ZONDERLAND et al., 2010) e comprometimento do bem-estar dos animais (HUNTER et al., 2001, SANDERCOCK et al., 2016). Nesse contexto, a única medida preventiva, amplamente recomendada por técnicos e utilizada em muitas granjas, é a caudectomia que consiste na amputação do terço final da cauda dos leitões, por meio de esmagamento, corte seguido (ou não) por cauterização (HUNTER et al., 2001). Mas seus benefícios ainda são controversos, pois surtos de caudofagia podem atingir até $60 \%$ dos animais, mesmo quando eles são submetidos à caudectomia (TEMPLE et al., 2011).

Ademais, uma alta ocorrência de lesões no aparelho mamário das porcas e na pele dos leitões, ocasionada por brigas durante a disputa por leite materno, tem sido descrita nos sistemas intensivos de produção. Isso pode ocorrer como resultado do aumento do número de leitões por leitegada, que vem ocorrendo nas últimas décadas, sem que o aumento de produção de leite das porcas ocorresse na mesma proporção (LE DIVIDICH, 2005; ELIASSON \& ISBERG, 2011). Em concordância com essa teoria, GALLoIs et al. (2005) reportaram que a frequência e a gravidade das lesões no aparelho mamário foram maiores nos tetos menos produtivos das matrizes. A incidência de lesões faciais nos leitões também é maior em leitegadas numerosas, em decorrência de maior disputa pelos tetos (FRASER, 1975).

Assim, o desgaste ou corte dos dentes dos leitões é uma prática de manejo que, apesar de já ter sido banida de algumas granjas, ainda pode ser considerada rotineira e utilizada para contornar as implicações da baixa disponibilidade de alimento para os leitões. Normalmente, o desgaste remove de 2 a 4 milímetros dos dentes, sendo realizado com pedra porosa rotativa (desgastador ou desbastador), que pode ser acoplada ao dispositivo de resfriamento com o uso de àgua. Já o corte dos dentes é realizado com alicate e consiste no corte na base do terço superior do dente ou bem próximo à gengiva. Apesar dos benefícios esperados, a prática do corte dos dentes também possui eficácia controversa sobre a redução das lesões no aparelho mamário das matrizes e na melhoria do desempenho dos leitões (ESTIENNE et al., 2001; SELEGATTo et al., 2003; SOUZA et al., 2004; KolLER, 2006). Desde 2001, a legislação da União Europeia proíbe a prática do desgaste ou corte dos dentes dos leitões como manejo de rotina, deixando a critério do Médico Veterinário indicar sua necessidade somente quando houver indícios de ferimentos nos tetos das matrizes, protegendo assim a saúde e o bem-estar animal (EC, 2001).

Considerando o modelo dos "Cinco Domínios" do bem-estar animal, proposto por MELLOR \& REID (1994), a submissão do leitão à caudectomia e ao desgaste ou corte dos dentes (Figura 3) podem ser considerados um desafio à saúde dos animais (Domínio 3 - Saúde). A caudectomia ocasiona um processo inflamatório que pode durar até 41dias após o procedimento (KENT et al., 2000; SUTHERLAND et al., 2008, 2011). Por sua vez, o corte ou desgaste dos den- 


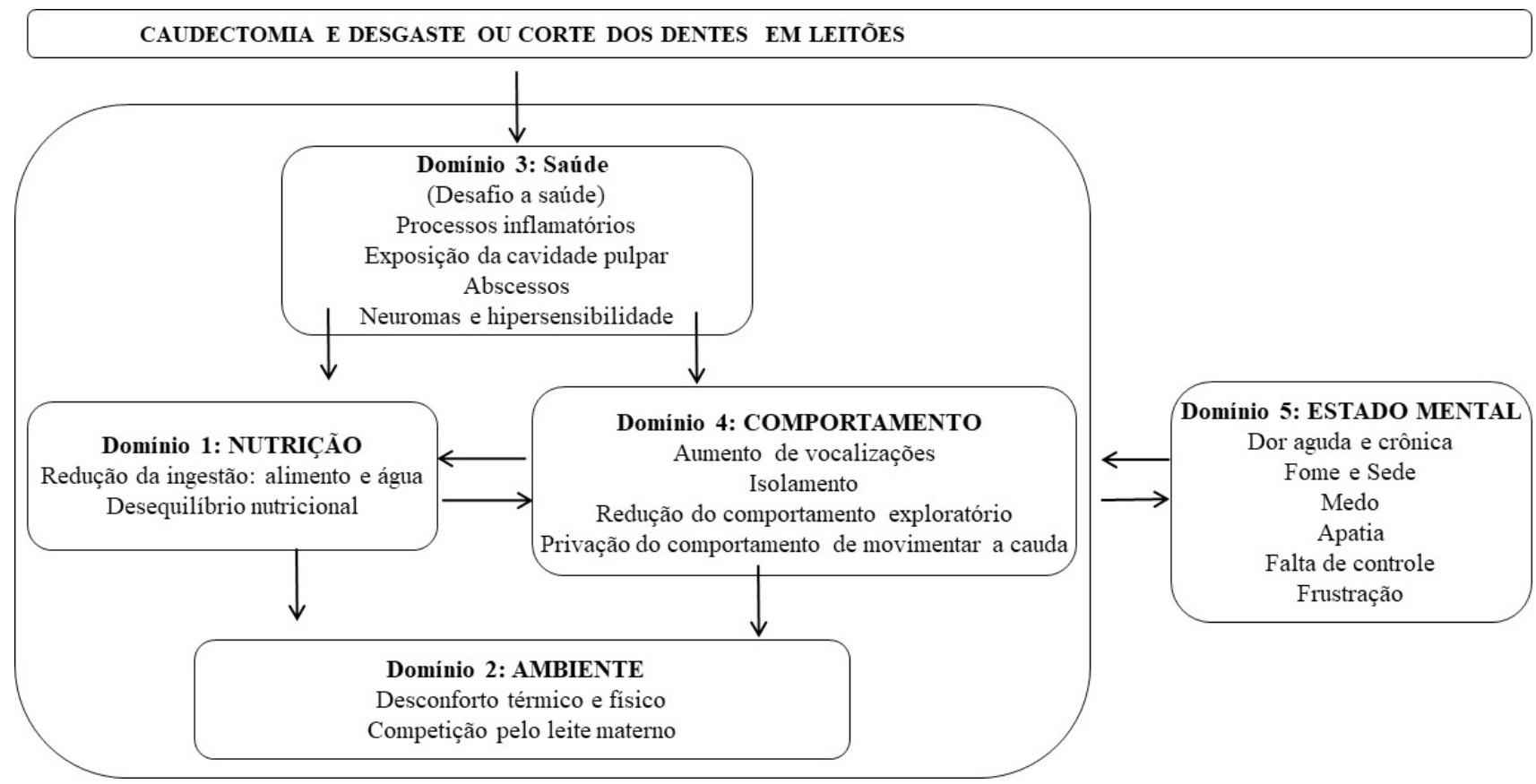

Figura 3. Aplicação do modelo "Cinco Domínios", proposto por MELLOR \& REID (1994) para a avaliação do impacto dos procedimentos de caudectomia e desgaste ou corte dos dentes sobre o bem-estar de leitões, baseando-se na literatura científica.

tes são fatores de risco para a exposição da cavidade pulpar, que resulta em gengivites, cáries, inflamações da polpa dentária e abscessos (SELEGATTO et al., 2003; KOLLER, 2006). Tanto a caudectomia quanto o desgaste ou corte dos dentes dos leitões geralmente são realizados sem anestesia e/ou analgesia, o que resulta em dor aguda, que pode se tornar crônica, dependendo das intercorrências após os procedimentos (NANNONI et al., 2014; SUTHERLAND, 2015; IsON et al., 2016). SANDERCOCK et al. (2016) comprovaram que há formação de neuromas traumáticos, em decorrência dos processos de reparação dos nervos periféricos lesionados, até quatro meses após a caudectomia em leitões. Adicionalmente, há evidências de hipersensibilidade periférica da região lesionada, o que se assemelha a caracterís- ticas de dor neuropática relatadas em humanos, conhecida como dor "fantasma", gerando sensação de dor crônica nesses animais (NANNONI et al., 2014; GIMINIANI et al., 2017a).

Destaca-se que a sensação de dor pode alterar o comportamento dos animais (Domínio 4 - Comportamento), resultando no aumento da vocalização durante a realização dos procedimentos (GIMINIANI et al., 2017b). Leitões que foram submetidos ao corte da cauda e dos dentes foram observados sozinhos com maior frequência durante os três primeiros dias após o procedimento e durante todo o período de amamentação (ZHOU et al., 2013). Considerando que nessa fase é comum os leitões se aglomerarem para manter a temperatura corporal, esse comportamento de isolamento poderia tornar os animais 
ainda mais susceptíveis a hipotermia, comprometendo seu conforto térmico e, em casos mais graves, sua sobrevivência.

Ainda, leitões submetidos à caudectomia e ao corte dos dentes apresentaram menor frequência de comportamentos exploratórios e de investigação do ambiente durante os períodos de creche e de crescimento (ZHOU et al., 2013). Essa modificação de comportamento poderia dificultar a localização de recursos, como água e alimento, em novo ambiente. Adicionalmente, animais que experimentam a sensação de dor reduzem a ingestão de alimentos e água, ocasionando sensações de fome e sede, que ao longo do tempo poderão resultar em casos de desnutrição (Domínio 1 - Nutrição). De fato, apesar de não terem avaliado o consumo dos animais, alguns estudos mostraram menor ganho de peso em suínos que passaram por caudectomia, nas primeiras 24 a 48 horas (MARCHANT-FORDE et al., 2009) e até 70 dias (ZHOU et al. 2013) após o procedimento. Existe ainda outra maneira pela qual a caudectomia tem o potencial de afetar o comportamento dos animais, que é através do uso da cauda como um mecanismo de comunicação entre os membros do grupo, tornando-os impossibilitados de comunicar-se por essa via (HOUPT, 2005). Portanto, o corte da cauda impede os animais de apresentarem este comportamento natural da espécie (NANNONI et al., 2014).

Conforme apresentado, todos os 4 domínios físicos afetados por esses procedimentos dolorosos em leitões resultam no comprometimento do Domínio 5 - Estado mental. Segundo
NoONAN et al. (1994), a contenção do leitão para realização dos procedimentos de caudectomia e corte dos dentes é estressante por si só. De fato, procedimentos dolorosos onde os animais precisam ser contidos fazem com que, além da sensação de dor, eles experimentem sensações de desconforto, medo, desconfiança e falta de senso de controle sobre a própria vida. Adicionalmente, a vivência dessas sensações pode levar o animal a estados de apatia e isolamento, afetando diretamente o Domínio 5 - Estado Mental.

Diante do exposto, fica claro que a realização da caudectomia e do desgaste ou corte dos dentes não resolve as causas que levam à ocorrência da caudofagia e das lesões na pele dos leitões ou no aparelho mamário das matrizes, apenas amenizam os efeitos desses comportamentos. Além disso, parece pouco coerente submeter um animal a um procedimento que Ihe causa dor aguda e pode, inclusive, lhe causar dor e desconforto crônico, como forma de evitar uma possível dor no futuro em decorrência da caudofagia e de lesões na pele, por exemplo. Nesse contexto, existe uma lacuna no que se refere a alternativas verdadeiramente eficientes para resolver esses problemas, os quais têm consequências negativas ao longo da vida dos animais, comprometendo seu bem-estar. Portanto, são necessárias estratégias de mitigação dessas consequências sobre os animais e alternativas e práticas de gestão que eliminem a necessidade de executar esses procedimentos dolorosos. $\mathrm{O}$ enriquecimento ambiental com palha nas baias de terminação (SCollo et al., 2013), boa venti- 
lação e espaço duplo nos comedouros (HUNTER et al., 2001) por exemplo, foram eficientes para diminuir a caudofagia em suínos. Além disso, o uso de analgesia e anestesia local pode reduzir acentuadamente a dor durante e após a realização dos procedimentos dolorosos (SUTHERLAND, 2011, 2015).

\section{APLICAÇÃo do MOdelo "CinCo DomínIOS" PARA} AVALIAÇÃO DO BEM-ESTAR DE FRANGOS DE CORTE SUBMETIDOS AO CARREGAMENTO E TRANSPORTE PARA O ABATE EM CAIXAS COM ALTA DENSIDADE

O abate de frangos de corte no Brasil aumentou nos últimos anos e já ultrapassou a marca de cinco bilhões de aves abatidas anualmente, posicionando o país entre os três maiores produtores mundiais (IBGE, 2017). Grande disponibilidade de área para construção de instalações e produção de grãos, solo fértil, clima favorável e inovação das empresas envolvidas no processo foram fatores que contribuíram para o crescimento do setor nos últimos dez anos (OLIVEIRA \& NÄÄs, 2012).

O processo de produção de frangos é extremamente complexo e, na maior parte do país, ocorre em sistema de integração. Segundo a Lei no 13.288 de 16 de maio de 2016 (BRASIL, 2016), integração é definida como a "relação contratual entre produtores integrados e integradores que visa planejar e realizar a produção e a industrialização ou comercialização de matéria-prima, bens intermediários ou bens de consumo final, com responsabilidades e obrigações recíprocas estabelecidas em contratos de integração". No caso da avicultura, os contratos geralmente dividem obrigações sendo os avicultores responsáveis pelos custos do aviário, da mão-de-obra e dos equipamentos e a empresa integradora responsável pelo fornecimento dos animais, ração, medicamentos, assistência técnica e, em alguns casos, pelo transporte das aves ao frigorífico (ZALUSKI \& MARQUES, 2015). Todas as etapas da criação de frangos (desde o nascimento até o abate) exigem boa logística e bom planejamento.

Do ponto de vista econômico, o transporte faz parte da gestão estratégica e representa $60 \%$ dos custos logísticos, sendo diretamente afetada pelo planejamento dos carregamentos, programação dos veículos, roteirização, auditoria de fretes e gerenciamento de avarias (FLEURY, 2002). Adicionalmente, o período do dia em que as aves são transportadas, as condições das estradas, o tempo de transporte e de espera no frigorífico para o abate bem como o número de aves nas caixas são fatores reconhecidamente capazes de afetar a mortalidade das aves (VIEIRA et al., 2013). Apesar disso, como estratégia errônea para reduzir os custos com a logística, observa-se em algumas situações o aumento do número de aves nas caixas (alta densidade), comprometendo o bem-estar animal.

Para definir alta densidade no transporte das aves serão considerados os valores que ultrapassam o definido pela legislação europeia, relativa à proteção dos animais durante o transporte (EC, 2005), já que o Brasil não dispõe de nenhuma legislação específica sobre esse as- 
sunto. A legislação europeia determina um espaçamento entre 180 e $200 \mathrm{~cm}^{2} / \mathrm{kg}$ para aves com peso vivo menor que $1,6 \mathrm{~kg} ; 160 \mathrm{~cm}^{2} / \mathrm{kg}$ para aves com peso vivo entre 1,6 e $3,0 \mathrm{~kg} ; 115 \mathrm{~cm}^{2} / \mathrm{kg}$ para aves entre 3,0 e 5,0 kg e $105 \mathrm{~cm}^{2} / \mathrm{kg}$ para aves com mais de 5,0 kg de peso vivo. Apesar desses valores definidos, é sabido que os mesmos podem variar em função do estado físico das aves e das condições climáticas, por exemplo. Para a presente abordagem, essas variações serão desconsideradas, em função da complexidade e especificidades de seus efeitos, dificultando sua inclusão no modelo geral proposto.

Considerando o modelo dos "Cinco Domínios" do bem-estar animal, proposto por MELLOR \& REID (1994), a alta densidade das caixas transportadoras de frangos de corte para abate (Figura 4) pode ser, inicialmente, classifi- cada como um desafio ambiental (Domínio 2 Ambiente), provocando aumento da temperatura local e corporal da aves, resultando em maior estresse calórico (DELEZIE et al., 2007). Uma ave produz cerca de 10 a 15 watts de calor com perda de 10,5 g de água por hora, assim, quanto maior o número de aves juntas em espaço reduzido, maior o incremento de calor e de umidade (Mitchell \& KetTlewell, 1998). A ave, por ser homeotérmica, em condições de mudança da temperatura ambiental, tentará regular sua temperatura corporal. Para isso ocorrerão algumas alterações fisiológicas e comportamentais, que podem afetar o Domínio 3 - Saúde e Domínio 4 Comportamento.

Nessas condições, a ave irá eriçar as penas e tentará expor a maior área de superfície corporal abrindo as asas. Concomitantemente ALTA DENSIDADE DE AVES NAS CAIXAS TRANSPORTADORAS

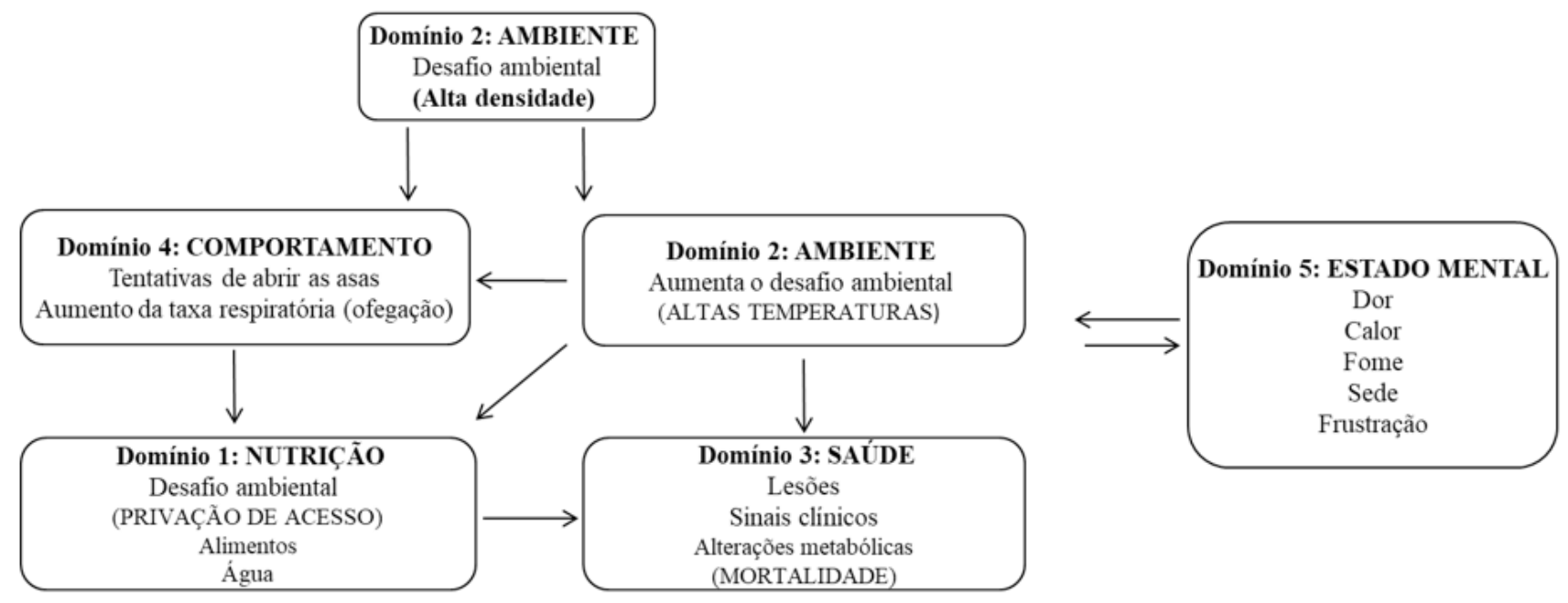

Figura 4. Aplicação do modelo "Cinco Domínios", proposto por MELLOR \& REID (1994) para a avaliação do impacto da alta densidade de aves nas caixas transportadoras, baseando-se na literatura científica. 
ocorrerá aumento da frequência respiratória, facilmente visualizada por meio da taxa de ofegação, que por sua vez poderá provocar alteração do equilíbrio ácido-base, gerando uma alcalose respiratória, devido ao estresse calórico (BORGES et al., 2003). A exposição das aves por 15 minutos a uma temperatura ambiental de $42^{\circ} \mathrm{C}$ já é suficiente para afetar o metabolismo proteico de frangos em crescimento, provocando redução significativa dos aminoácidos livres circulantes no plasma, proteínas totais e da concentração da enzima alcalina fosfatase, além da elevação das concentrações de certos grupos de aminoácidos no fígado, albumina, glicose, ácido úrico, ácidos graxos livres no plasma, lactato desidrogenase e creatina quinase (OSTROWSKI-MEISSNER, 1981). O aumento da temperatura está diretamente relacionado com o aumento da síntese da proteína de choque térmico, responsável por proteger as células em situações de estresse (DELEZIE et al., 2007).

Na granja, o aumento da temperatura também promoveria uma elevação no consumo de água e a ingestão da bebida fria auxiliaria na redução da taxa respiratória, na perda de calor e no reestabelecimento da homeostase (BoRGES et al., 2003). Entretanto, dentro das caixas, as aves não possuem acesso a água (Domínio 1 - Nutrição) e o espaço interno é restrito, impossibilitando que as aves estendam as asas (Domínio 4 - Comportamento), fatores que irão dificultar o processo de perda de calor. A impossibilidade de beber água promoverá um estado de desconforto causando sede e, com o passar do tempo, pode levar o animal à desidratação (VANDERHASSELT et al., 2013). Além disso, a permanência prolongada das aves nessas condições de restrição de espaço impossibilita a execução de comportamentos específicos, como os de manutenção, gerando estados emocionais negativos, como frustração (Domínio 5 - Estado mental) (VESTERGAARD et al., 1997).

Com o passar das horas confinadas nesse ambiente, as aves sentirão necessidade de ingerir alimento. Segundo Rul et al. (2011), aves em condições normais se alimentam a cada 4 horas, sendo assim, períodos prolongados sem acesso a alimento (Domínio 1 - Nutrição) alteram o comportamento das aves que passam a frequentar mais os bebedouros, ingerem mais água e podem iniciar o consumo de cama do aviário (Domínio 4 - Comportamento). Casos extremos de privação de alimento podem levar a estados mentais negativos caracterizados pela fome e frustração (DE JONG et al., 2002). Todavia a retirada da ração para o transporte é fundamental, pois evita problemas de contaminação no frigorífico durante o abate, que pode ocorrer devido à presença de alimento no trato gastrointestinal (NORTHCUTT et al., 1997). Neste caso é importante seguir a recomendação brasileira, a qual estabelece que o jejum para o abate não deve ultrapassar um total de 12 horas (RosA et al., 2013).

Dentro do contexto apresentado, todos os 4 domínios físicos afetados pela alta densidade no carregamento e no transporte de aves resultam no comprometimento do Domínio 5 - Estado Mental e, quando não resolvidos a 
tempo, podem acarretar em sério comprometimento do bem-estar animal, da qualidade de carne e, em casos extremos, na morte das aves. Encontrar um ponto de equilíbrio entre bem-estar animal e os aspectos econômicos do processo de produção é fundamental para garantir um produto ético, rentável e de alta qualidade.

\section{CONCLUSÃo}

O modelo dos "Cinco Domínios" do bem-estar animal disponibiliza uma visão integrada sobre os riscos de comprometimento dos domínios da nutrição, do ambiente, da saúde, do comportamento e do estado mental dos animais, em situações que envolvem restrição de espaço em confinamentos de bovinos, procedimentos dolorosos, incluindo a caudectomia e o corte dos dentes em leitões e o carregamento e transporte de frangos de corte em altas densidades. Apesar de não ser possível incluir todos os fatores intervenientes na dinâmica dos sistemas produtivos e suas possíveis combinações, é notório o risco de comprometimento do bem-estar animal nas situações aqui descritas.

\section{REFERÊNCIAS}

AWIN (Animal Welfare Indicators). 2015a. AWIN Welfare Assessment Protocol for Goat. Disponível em: <https://air.unimi.it/retrieve/ handle/2434/269102/384790/AWINProtocolGoats.pdf>. Acesso em: 18 abr. 2018.

AWIN (Animal Welfare Indicators). 2015b. AWIN Welfare Assessment Protocol for Sheep. Disponível em: <https://air.unimi.it/re- trieve/handle/2434/269097/384836/ AWINProtocolSheep.pdf>. Acesso em: 18 abr. 2018.

BeAusoleil, N.J. \& MelloR, D.J. 2015. Advantages and limitations of the 'Five Domains' model for assessing animal welfare impacts associated with vertebrate pest control. New Zealand Veterinary Journal 63 (1): 37-43.

BeAusoleil, N.J.; Fisher, P.; MelLoR, D.J. \& WARBURTON, B. 2012. Ranking the negative impacts of wildlife control methods may help advance the three Rs. Alternatives to Animal Experimentation Proceeding I (WC8): 481-485.

BLOKHUIS, H.J.; JONeS, R.B.; Geers, R.; Miele, M. \& VEISSIER, I. 2003. Measuring and monitoring animal welfare: Transparency in the food product quality chain. Animal Welfare 12 (4): 445-455, 2003.

Blokhuis, H.J.; Keeling, L.J.; Gavinelli, A. \& SeRRATOSA, J. 2008. Animal welfare's impact on the food chain. Trends in Food Science \& Technology 19 (1): S75-S83.

Borges, S.A.; MAIORKA, A. \& Fisher DA Silva, A.V. 2003. Fisiologia do estresse calórico e a utilização de eletrólitos em frangos de corte. Ciência Rural 33 (5): 975-98.

BRASIL. 2016. Presidência da República - Casa Civil. Lei no 13.288, de 16 de maio de 2016 . Diário Oficial da União, Brasília, DF, 17 de maio de 2016. Disponível em:< http:// 
www.planalto.gov.br/ccivil_03/_ato20152018/2016/lei/l13288.htm>. Acesso em: 18 abr. 2018.

BROOM, D.M. Indicators of poor welfare. 1986. The British Veterinary Journal 142 (6): 524-526.

BRoOM, D.M. \& FrASER, A.F. 2007. Domestic animal behaviour and welfare. Wallingford, CAB Publising, IV, 540p.

Campbell, J.B.; Catangui, M.A.; Thomas, G.D.; BOXLER, D.J. \& DAVIS, R. 2001. Effects of stable flies (diptera: Muscidae) and heat stress on weight gains of grazing yearling cattle. Journal of Economical Entomology 94 (3): 780-783.

CARROLL, J.A. \& ForSBERG, N.E. 2007. Influence of stress and nutrition on cattle immunity. Veterinary Clinics of North America: Food Animal Practice 23 (1): 105-149.

Catangui, M.A.; CAMPBell, J.B.; Thomas, G.D. \& BOXLER, D.J. 1997. Calculating economic injury levels for stable flies (diptera: Muscidae) on feeder heifers. Journal of Economical Entomology 90 (1): 6-10.

DE JONG, I.C.; VOORST, S.V.; EHLHARDT, D.A. \& BLOKHUIS, H.J. 2002. Effects of restricted feeding on physiological stress parameters in growing broiler breeders. British Poultry Science 43 (2): 157-168.

DE PASSILLÉ, A.M. \& RUSHEN, J. 2005. Food safety and environmental issues in animal wel- fare. Review of Science and Technology off International Epizooties 24 (2): 757-766, 2005.

Delezie, E.; SWennen, Q.; Buyse, J. \& Decuypere, E. 2007. The effect of feed withdrawal and crating density in transit on metabolism and meat quality of broilers at slaughter weight. Poultry Science 86 (7): 1414-1423.

EC (European Comission). 2001. Directiva da Comissão, de 9 de Novembro de 2001, que altera a Directiva 91/630/CEE do Conselho relativa às normas mínimas de protecção de suínos. Disponível em: <https://eur-lex.europa.eu/legal-content/PT/TXT/HTML/?uri=CELEX:32001L0093\&from=en >. Acesso em: 18 abr. 2018.

EC (EUROPEAN COMmission). 2005. Regulamento $n^{\circ} 1 / 2005$ do Conselho de 22 de dezembro de 2004 relativo à protecção dos animais durante o transporte e operações afins e que altera as Directivas 64/432/CEE e 93/119/CE e o Regulamento $n^{\circ}$. 1255/97, 2005. Disponível em:<http://eur-lex.europa.eu/legal-content/PT/TXT/HTML/?uri=CELEX:32005R0001\&from=EN. Acesso em: 18 abr. 2018.

EliAsSON, C. \& ISBERG, S. 2011. Production and composition of sow milk. Disponível em: < https://stud.epsilon.slu.se/3754/1/eliasson_et_al_111231.pdf $>$. Acesso em: 18 abr. 2018.

Estienne M.J.; HoRsley B.R.\& HARPeR A.F. 2001. 
Case study: effects of pig needle teeth on pig and sow injuries and pre-weaning pig performance. The Professional Animal Scientist 1 (19): 68-71.

FELL, L.R.; COLDITZ, I.G.; WALKER, K.H. \& WATSON, D.L. 1999. Associations between temperament, performance and immune function in cattle entering a commercial feedlot. Animal Production Science 39 (7): 795-802.

FeLL, L.R.; WALKER, K.H.; REDdACLIFF, L.A.; DAVIES, L.; VAllance, H.J.; HOUSE, J.R. \& Wilson, S.C. 1998. Effects of yard weaning and pre-feedlot vaccination on feedlot performance of Bos taurus steers. Animal Production Australia 22: 173-176.

Fisher A.D.; SteWARt M.; Verkerk G.A.; MorRow C.J. \& MATTHEWS L.R. 2003.The effects of surface type on lying behaviour and stress responses of dairy cows during periodic weather induced removal from pasture. Applied Animal Behaviour Science 81 (1): 1-11.

FISHER, A.D.; CroWe, M.A.; O'KIELY, P. \& ENRIGHT, W.J. 1997. Growth, behavior, adrenal and immune responses of finishing beef heifers housed on slatted floors at 1 S, 2.0, 2.5 or $3.0 \mathrm{~m}^{2}$ space allowance. LivestockProduction Science 51 (1-3): 245-254.

FLEURY, P.F. 2002. Gestão estratégica do transporte. Revista Tecnologística (82): 60-67.

FrASER, D. 1975. The "teat order" of suckling pigs: Il. Fighting during suckling and the ef- fects of clipping the eye teeth. Journal of Agricultural Science 84 (3): 393 - 399.

FrASER, A.F. 1980. Farm animal behavior. Londres, Ballière Tindall, II, 196p.

FRASER, A.F. 1983. The behavior of maintenance and the intensive husbandry of cattle, sheep and pigs. Agriculture, Ecosystems \& Environment 9 (1): 1-23.

Gallois, M.; Le Cozler, Y. \& Prunier, A. 2005. Influence of tooth resection in piglets on welfare and performance. Preventive Veterinary Medicine 69 (1-2): 13-23.

Garnett, T.; Appleby. M.C.; Balmford, A.; BATEMAN, I.J.; Benton, T.G.; BloOMERS, P.; Burlingame, M.; DAWKINS, M.; Dolan, L.; Fraser, D.; Herrero, M.; Hoffman, I.; SMITH, P.; THORNTON, P.K.; TOULMIN, C.; Vermeulen, S.J. \& GodfraY, H.C.J. 2013. Sustainable intensification in agriculture: premises and policies. Science 341 (6141): 33-34.

GIMINIANI, P.D.; EDWARdS, S.A.; MALCOLM, E.M.; LEACH, M.C.; HERSKIN, M.S. \& SANDERCOCK, D.A. 2017a. Characterization of short- and long-term mechanical sensitisation following surgical tail amputation in pigs. Scientific Reports 7: 4827.

GIMINIANI, P.D.; NASIRAHMADI, A.; MalColm, E.M.; LEACH, M.C. \& EDWARDS, S.A. 2017b. Docking piglet tails: How much does it hurt and for how long? Physiology \& Behavior 182 (1): 69-76. 
GRANDIN, T. 2016. Evaluation of the welfare of cattle housed in outdoor feedlot pens. Veterinary and Animal Science 1 (1-2): 23-28.

Gupta, S.; Earley, B. \& Crowe, M.A. 2007. Pituitary, adrenal, immune and performance responses of mature Holstein x Friesian bulls housed on slatted floors at various space allowances. The Veterinary Journal 173 (3):594-604.

GygAX, L.; SIEgWART, R. \& WeChSLER, B. 2007. Effects of space allowance on the behavior and cleanliness of finishing bulls kept in pens with fully slatted rubber coated flooring. Applied Animal Behaviour Science 107 (1): 1-12.

Hickey, M.C.; EARLey, B. \& Fisher, A.D. 2003. The effect of floor type and space allowance on welfare indicators of finishing steers. Irish Journal of Agriculture and Food Research 42 (1): 89-100.

HOUPT, K.A. 2005. Domestic animal behavior for veterinarians and animal scientists. Ames, Wiley-Blackwell Publisher, IV, 416p.

Hunter, E.J.; JONES, T.A.; GUISE, H.J.; PENNY, R.H.C. \& HOSTE, S. 2001. The relationship between tail biting in pigs, docking procedure and other management practices. The Veterinary Journal 161 (1): 72-79.

IBGE (Instituto Brasileiro de Geografia e Estatística). 2017. Estatística da Produção Pecuária Junho de 2017. Disponível em: < http:// ftp.ibge.gov.br/Producao_Pecuaria/Fas-
ciculo_Indicadores_IBGE/abate-leite-couro-ovos_201701caderno.pdf $>$. Acesso em: 18 abr. 2018.

INGVARTSEN, K.L. \& ANDERSEN, H.R. 1993. Space allowance and type of housingfor growing cattle. A review of performance and possible relation to neuroendocrine function. Acta Agriculturae Scandinavica A - Animal Science 43 (2): 65-80.

ISON, S.H.; CLUTTON, R.E.; DI GIMINIANI. P. \& RUTHERFORD, K.M.D. 2016. A review of pain assessment in pigs. Frontiers in Veterinary Science 3: 1-16

KENT, J.E.; JACKSON, R.E.; MOLONY, V. \& HOSIE, B.D. 2000 Effects of acute pain reduction methods on the chronic inflammatory lesions and behaviour of lambs castrated and tail docked with rubber rings at less than two days of age. The Veterinary Journal 160 (1): 33-41.

KOLLER, F.L. 2006. Manejo dentário em leitões: efeitos no ganho de peso na maternidade e creche,prevalência de abscessos periapicais e isolamento dos agentes bacterianos envolvidos. Dissertação de mestrado, Universidade Federal do Rio Grande do Sul, Porto Alegre, Rio Grande do Sul, 55p.

Kondo, S.; Sekine, J.; OKubo, M. \& AsAhidA, Y. 1989. The effect of group size and space allowance on the agonistic and spacing behavior of cattle. Applied Animal Behaviour Science 24 (1-2): 127-135. 
KRAWCZEL, P.D.; KLAIBER, L.B.; ButZleR, R.E.; KLAIBER, L.M.; DANN, H.M.; MOONEY, C.S. \& GRANT, R.J. 2012. Short-term increases in stocking density affect the lying and social behavior, but not the productivity, of lactating Holstein dairy cows. Journal of Dairy Science 95 (8): 4298-4308.

KRITAS, S.M. \& MORRISON R.B. 2007. Relationships between tail biting in pigs and disease lesions and condemnations at slaughter. The Veterinary Record 160 (5): 149-152.

LEE, C.; Fisher, A.D.; ColditZ, I.G.; LEA, J.M. \& FERguson. D.M. 2013. Preference of beef cattle for feedlot or pasture environments. Applied Animal Behaviour Science 145 (3): 53-59.

LE DIVIDICH, J.; ROOKE, J. A. \& HERPIN, P. 2005. Nutritional and immunological importance of colostrum for the new-born pig. Journal of Agricultural Science 143 (6): 469-485.

LINDBERG, A.C. 2001. Group life, pp.37-54. In: KeEling, L.J.; Gonyou, H.W(eds.). Social Behavior in Farm Animals. Wallingford, CABI Publishing, 406p.

MACITELLI, F. 2015. Implicações da disponibilidade de espaço no confinamento de bovinos de corte. Tese de doutorado, Universidade Estadual Paulista "Júlio de Mesquita Filho", Jaboticabal, São Paulo, 90p.

Mader, T.L. 2008. Profit Tip: Managing Cattle Facilities To Minimize Mud. Disponível em: < https://beef.unl.edu/stories/200809111. shtml>. Acesso em: 18 abr. 2018.

MADER, T.L. 2003. Environmental stress in confined beef cattle. Journal of Animal Science 81 (2): E110-E119.

MARCHANT-FORDE, J.N.; LAY, D.C.; JR., MCMUNN, K.A.; Cheng, H.W.; Pajor, E.A. \& MARChANT-FordE, R.M. 2009. Postnatal piglet husbandry practices and well-being: the effects of alternative techniques delivered separately. Journal of Animal Science 87(4):1479-1492.

MAtTERI, R.L.; CARroll, J.A. \& DYer, C.J. 2000. Neuroendocrine Responses to Stress, pp. 269-290. In: MoRBEG, J.P. \& MENCH, J.A. (eds.). The Biology of Animal Stress: Basic Principles and Implications for Animal Welfare. New York, CABI Publishing, 392p.

Mellor, D.J.; PATterson-Kane, E. \& StafFord, K.J. 2009. The sciences of animal welfare. Oxford, Wiley-Blackwell, I, 224p.

MELLOR, D.J. \& REID, C.S.W. 1994. Concepts of animal well-being and predicting the impact of procedures on experimental animals. Disponível em: <http://org.uib.no/dyreavd/harm-benefit/Concepts\%20of\%20animal\%20well-being\%20and\%20predicting. pdf>. Acesso em: 18 abr. 2018.

MELLOR, D.J. 2016. Updating animal welfare thinking: Moving beyond the "Five Freedoms" towards "a Life Worth Living". Animals 6 (3): 21. 
MeLLOR, D.J. 2017. Operational Details of the Five Domains Model and Its Key Applications to the Assessment and Management of Animal Welfare. Animals 7(8): 60.

Mellor, D.J. \& BeAusoleil, N.J. 2015. Extending the 'Five Domains' model for animal welfare assessment to incorporate positive welfare states. Animal Welfare 24: 241-253.

Millen, D.D.; PACHECO, R. D.L.; ARRIGONI, M.D.B.; GALYEAN, M.L. \& VASCONCELOS, J.T. 2009. A snapshot of management practices and nutritional recommendations used by feedlot nutritionists in Brazil. Journal of Animal Science 87 (10): 3427-3439.

Mitchell, M.A., \& Kettlewell, P.J. (1998). Physiological stress and welfare of broiler chickens in transit: solutions not problems! Poultry science 77 (12): 1803-1814.

Mormède, P.; ANDANSON, S.; AUPÉRIN, B.; BeERDA, B.; GuÉmenÉ, D.; MALMKVIST, J.; MANTECA, X.; Manteuffel, G.; Prunet, P.; VAn ReENEN C.G.; RICHARD, S. \& VeISSIER, I. 2007. Exploration of the hypothalamic-pituitaryadrenal function as a tool to evaluate animal welfare. Physiology \& Behavior 92 (3): 317-339.

NANNONI, E.; VAlSAmi, T.; SARDi, L. \& Martelli, G. 2014. Tail docking in pigs: a review on its short- and long-term consequences and effectiveness in preventing tail biting. Italian Journal of Animal Science 13 (1): 98-106.
Napolitano, F.; de Rosab, G.; Grassob, F.; PACELLIA, C. \& BORDIB, A. 2004. Influence of space allowance on the welfare of weaned buffalo (Bubalus bubalis) calves. Livestock Production Science 86 (1): 117-124.

NoOnAN, G.J.; RAND, J.S.; PRIEST, J.; AInSCOW, J. \& BLACKSHAW, J.K. 1994. Behavioural observations of piglets undergoing tail docking, teeth clipping and ear notching. Applied Animal Behaviour Science 39 (3-4): 203213.

NorthCutT, J.K.; SAVAge, S.I. \& VeSt, L.R. 1997. Relationship between feed withdrawal and viscera condition of broilers. Poultry Science 76 (2): 410-414.

NRC (National Research Council). 1996. Nutrient requirements of beef cattle. Washington, The National Academies Press, VII, 242p.

OIE (World Organization for ANimal Health). 2013. Terrestrial Animal Health Code. Disponível em: <http://www.oie.int/index. php?id=169\&L=0\&htmfile=chapitre_aw_ introduction.htm>. Acesso em: 18 abr. 2018.

OliveirA, C.A. \& MilLEN, D.D. 2014. Survey of the nutritional recommendations and management practices adopted by feedlot cattle nutritionists in Brazil. Animal Feed Science and Technology 197: 64-75.

OLIVEIRA, D.R.M.S. \& NÄÄS, I.A. 2012. Issues of sustainability on the Brazilian broiler meat 
production chain. Disponível em: <http:// ainfo.cnptia.embrapa.br/digital/bitstream/item/72778/1/Issues.pdf>. Acesso em 18 abr. 2018.

OSTROWSKI-MEISSNER, H.T. (1981). The physiological and biochemical responses of broilers exposed to short-term thermal stress. Comparative Biochemistry and Physiology Part A: Physiology 70 (1): 1-8.

PARANhos dA COSTA, M.J.R. 2000. Ambiência na produção de bovinos de corte. In: XVIII Encontro Anual de Etologia, Florianópolis, Brasil, p.1-15.

Pelletier, N.; Pirog, R. \& Rasmussen, R. 2010. Comparative life cycle environmental impacts of three beef production strategies in the Upper Midwestern United States. Agricultural Systems 103 (6): 380-389.

PHILLIPS, C. 2008. Cattle behaviour and welfare. Cambridge, Blackwell Science, II, 274p.

RASMUSSEN, R.; Fontes, A. \& CORDINGLEY, B. 2014. Beefing up in Brazil - Feedlots to drive industry growth. Disponível em: <https://www.beefcentral.com/wp-content/ uploads/2014/10/Rabobank_IN459_Beefing_up_in_Brazil.pdf>. Acesso em: 18 abr. 2018.

Rosa, P. S., Albino, J. J., BAsSi, L. J., Grah, R. A. \& NIENDICCKER, T. P. 2013. Manejo pré-abate em frangos de corte. Embrapa Suínos e Aves (Recomendação Técnica). Disponível em: < https://ainfo.cnptia.embrapa.br/
digital/bitstream/item/78929/1/INSTRU-

CAO-36.pdf>. Acesso em: 18 abr. 2018.

RUI, B.R.; ANGRIMANI, D.D.S.R. \& SILVA, M.A.A.D. 2011. Pontos críticos no manejo pré-abate de frango de corte: jejum, captura, carregamento, transporte e tempo de espera no abatedouro. Ciência Rural 41(7): s/p.

RUSHEN, J. \& DE PASSILÉ, A.M.B. 1992. The scientific assessment of the impact of housing on animal welfare: a critical review. Canadian Journal of Animal Science 72 (4): 721-743.

SANDERCOCK, D.A.; SMITH, S.H.; GIMINIANI, P.D. \& EDWARDS, S.A. 2016. Histopathological characterization of tail injury and traumatic neuroma development after tail docking in piglets. Journal of Comparative Pathology 155 (1): 40-49.

SAPOLSKY, R.M. 2005. The influence of social hierarchy on primate health. Science 308 (5722), 648-652.

Scollo, A.; Martino, G.D.; Bonfanti, L.; Stefani, A.L.; SChiavon, E.; MARANGon, S. \& GotTARDO, F. 2013. Tail docking and the rearing of heavy pigs: The role played by gender and the presence of straw in the control of tail biting. Blood parameters, behaviour and skin lesions. Research in Veterinary Science 95 (2): 825-830.

SegabinazZI, L.R.; DE MenezeS, L.F.G.; DA Silva, C.E.K.; MARtinello, C.; Boito, B. \& MolineTE, M. L. 2014. Diurnal ingestive behavior of Holstein calves reared in different systems: 
feedlot or pasture. Acta Scientiarum: Animal Sciences 36 (2): 225-231.

Selegatto, M.A.; Carvalho, L.F.O.S; Oliveira, C. J.B \& DOMINGUES JR., F.J. 2003. Ocorrência de cáries em suínos de abate. In: XI Congresso Brasileiro de Veterinários Especialistas em Suínos (ABRAVES), Concórdia, Brasil, p. 157-158.

SOUZA, M.A.; SOBESTIANSKY, J.; LOPES, E.L.; COSTA, M.C. \& NUNES, R.C. 2004. Teeth clipping, grinding and unclipping: influence on sows and piglets injuries and performance of the piglets in a farm in Rio Verde - GO - Brazil. In: XVIII International Pig Veterinary Society Congress, Hamburgo, Alemanha, p. 795.

StOKKA, G.L.; LeCHTENBERG, K.;EdWARDS, T.;MACGregor, S.; Voss, K.; Griffin, D.; GroteLUesChen, D.M.; , R.A. \& Perino, L.J. 2001. Lameness in Feedlot Cattle. Veterinary Clinics: Food Animal Practice 17(1): 189207.

SUTHERLAND, M.A. 2015. Welfare implications of invasive piglet husbandry procedures, methods of alleviation and alternatives: a review. New Zealand Veterinary Journal 63 (1): 52-57.

SUtheRLAND, M.A.; DAVIS, B.L, \& McGLone, J.J. 2011. The effect of local or general anesthesia on the physiology and behavior of tail docked pigs. Animal 5 (8): 1237-1246.

Sutherland, M.A.; BRYER, P.J.; KReBS, N. \& MC GLONE, J.J. 2008. Tail docking in pigs: acute physiological and behavioural responses. Animal 2 (2): 292-297.

SWeeten, J.; Lubinue, L.; Durland, R. \& Bruce, B. 2014. Feedlot mounds - Beef Cattle Handbook. Disponível em: <http://www. iowabeefcenter.org/bch/FeedlotMounds. pdf>. Acesso em: 18 abr. 2018.

Temple, D.; Manteca, X.; Velarde, A. \& Dalmau, A. 2011. Assessment of animal welfare through behavioural parameters in Iberian pigs inintensive and extensive conditions. Applied Animal Behaviour Science 131 (12): 29-39.

Tsigos, C. \& Chrousos, G.P. 2002. Hypothalamic-pituitary-adrenal axis, neuroendocrine factors and stress. Journal of Psychosomatic Research 53 (4): 865-871.

VANDERHASSELt, R.F.;BUIJS, S.; SPRENGER, M.; GOETHALS, K.; Willemsen, H.;DuchateaU, L., \& TUYTTENS, F.A.M. (2013). Dehydration indicators for broiler chickens at slaughter. Poultry Science 92 (3): 612-619.

Vanhonacker, F.; Verbeke, W.; Van Poucke, E.; BUIJS, S. \&TUYTTENS, F.A.M. 2009. Societal concern related to stocking density, pen size and group size in farm animal production. Livestock Science 123 (1): 16-22.

VEISSIER, I. \& BOISSY, A. 2007. Stress and welfare: Two complementary concepts that are intrinsically related to the animal's point of view. Physiology \& Behavior 92 (3): 429433. 
VestergaArd, K.S.; Skadhauge, E.\& LAWSON, L.G. 1997. The stress of not being able to perform dustbathing in laying hens. Physiology \& Behavior 62(2): 413-419.

VERMEIR, I. \& VerbeKe, W. 2006. Sustainable food consumption: exploring the consumer "attitude-behavioral intention" gap. Journal of Agricultural and Environmental Ethics 19 (2): 169-184.

VIEIRA, F.M.C.; Silva, I.J.O.; BARBOSA FILHO, J.A.D. \& VIEIRA, A.M.C. 2013. Redução das perdas pré-abate de frangos de corte: efeito da densidade de aves submetidas aos diferentes tempos de espera no abatedouro. Journal of Animal Behaviour and Biometerology $1(1), 1-6$.

Welfare Quality ${ }^{\circledR}$ Consortium. 2009. Welfare quality $^{\circledR}$ assessment protocol for cattle. Lelystad, The Netherlands, I, 180p.

ZALUSKI, P.R.S \& MARQUES, I.C. 2015. Vantagens e desvantagens do sistema de integração vertical na avicultura de corte. In: XXXV Encontro Nacional de Engenharia de Producao Perspectivas Globais para a Engenharia de Produção, Fortaleza, Brasil. Disponível em: <http://www.abepro.org.br/biblioteca/TN_WIC_206_219_27184.pdf>. Aces- so 18 fev. 2018.

ZHOU, B.; YANG, X.J.; ZHAO, R.Q.; HUANG, R.H.; WANG, Y.H.; WANG, S.T.; YIN, C.P.; SHEN, Q.; WANG, L. Y. \& SCHINCKEL, A. P. 2013. Effects of tail docking and teeth clipping on the physiological responses, wounds, behavior, growth, and backfat depth of pigs. Journal of Animal Science 91 (10): 4908-4916.

ZONDERLAND, J.J.; BRACKE, M.B.M.; DEN HARTOG, L.A.; KeMP, B. \& SPOOLDER, H.A.M. 2010. Gender effects on tail damage development in single-or mixed-sex groups of weaned piglets. Livestock Science 129 (13): 151-158.
Recebido: 02/03/2018

Revisado: 20/04/2018

Aceito: 15/05/2018 